\title{
Nonlinear Response Properties of Combination-Sensitive Electrosensory Neurons in the Midbrain of Gymnarchus niloticus
}

\author{
Bruce A. Carlson and Masashi Kawasaki \\ Department of Biology, University of Virginia, Charlottesville, Virginia 22904
}

\begin{abstract}
The jamming avoidance response of the weakly electric fish Gymnarchus niloticus relies on determining the sign of the frequency difference (Df) between the fish's own electric organ discharge (EOD) and that of a neighbor, which is achieved by comparing modulations in amplitude (AM) and phase (PM) that result from the summation of their EODs. These two stimulus features are processed in separate pathways that converge in the torus semicircularis on combination-sensitive neurons, many of which are selective for the sign of Df. We recorded extracellular single-unit responses to independent stimulation with AM and PM and combined AM-PM stimulation to determine how sign selectivity is established. Responses to AM and PM frequently summated nonlinearly, leading to sign-selective responses as a result of facilitation to the preferred sign of Df and/or suppression to the nonpreferred sign of Df. Facilitation typically occurred when responses to AM and PM were aligned, whereas suppression typically occurred when they were offset. By experimentally manipulating the degree of alignment between these two responses, we found that the summed response was dependent on their relative timing. In addition, we found a unique class of units that were sensitive to differences in amplitude between two body surfaces. This sensitivity rendered such units immune to the problem of orientation ambiguity, in which the sign selectivity of a single neuron reverses with changes in stimulus orientation. We discuss potential synaptic mechanisms for driving nonlinear responses in these and other combination-sensitive neurons.
\end{abstract}

Key words: electric fish; mormyriform; facilitation; suppression; torus semicircularis; jamming avoidance response

\section{Introduction}

Combination-sensitive neurons play a critical role in sensory processing because they are specifically tuned to the cooccurrence of multiple stimulus features (Suga, 1994). To function as an ideal feature detector, a combination-sensitive neuron should behave like a neuronal AND gate that only responds if all stimulus features are present, as opposed to a neuronal OR gate that responds if any feature is present (Schnupp and King, 2001). Such a computational feat requires a nonlinear combination of responses, such that the presence of one feature enhances the response to the other, or conversely, the absence of one feature suppresses the response to the other. Several studies demonstrate that combinationsensitive neurons indeed process inputs nonlinearly (Fuzessery and Feng, 1983; Margoliash, 1983; Misawa and Suga, 2001; Peña and Konishi, 2001), and theoretical treatments have suggested several possible mechanisms (Koch and Poggio, 1992). However, we have a poor understanding of how combination-sensitive neurons actually achieve this type of

Received May 25, 2004; revised July 28, 2004; accepted July 30, 2004.

This work was supported by National Science Foundation Grants IBN-9974811 and IBN-0235533 to M.K.

Correspondence should be addressed to Bruce A. Carlson, Department of Biology, Gilmer Hall, P.0. Box 400328,

University of Virginia, Charlottesville, VA 22904-4328. E-mail: bc6s@virginia.edu.

DOI:10.1523/JNEUROSCI.2021-04.2004

Copyright $\odot 2004$ Society for Neuroscience $\quad$ 0270-6474/04/248039-10\$15.00/0 computation: how can the response to multiple stimulus parameters be different from the sum of responses to each?

The African fish Gymnarchus niloticus produces a continuous, quasisinusoidal electric organ discharge (EOD) for active electrolocation and communication (Lissman, 1958). Electrolocation is impaired by interference from a conspecific EOD of similar frequency (Heiligenberg, 1975). In response to such a "jamming" stimulus, an individual will shift its EOD frequency to increase the frequency difference and reduce interference, a behavior termed the jamming avoidance response (JAR) (Bullock et al., 1975). The decision to increase or decrease EOD frequency requires that the fish determine whether its frequency $\left(f_{1}\right)$ is higher or lower than its neighbor's frequency $\left(f_{2}\right)$, which is accomplished by analyzing modulations in amplitude (AM) and phase (PM) that result from combining the two signals (Kawasaki, 1993). Both AM and PM occur at frequencies equal to the magnitude of the frequency difference ( $\mathrm{Df}=f_{2}-f_{1}$ ) between the two EODs (Fig. 1A). Although AM and PM alone are identical for opposite signs of Df, their temporal relationship is reversed (Fig. $1 B-D)$. Thus, the sign of Df is determined by comparing AM with phase differences between different body surfaces [differential PM (DPM)].

In Gymnarchus, AM and DPM are processed in separate pathways within the medullary electrosensory lateral line lobe (Kawasaki and Guo, 1998). Both AM- and DPM-sensitive 

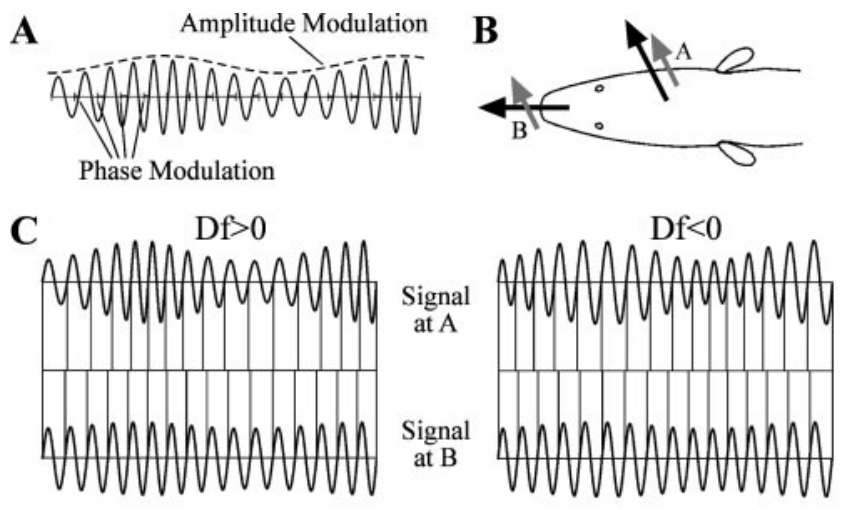

D
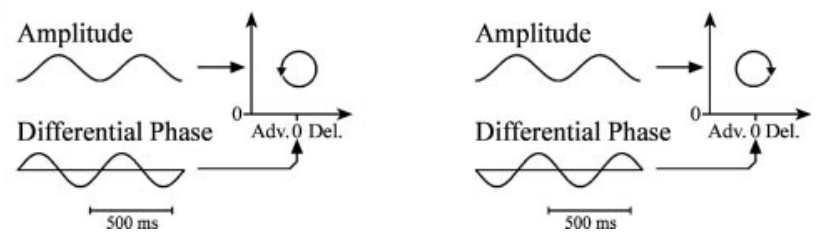

Figure 1. Sensory cues for the JAR. A, Combining two sinusoidal signals results in amplitude and phase modulation at a frequency equal to the frequency difference between the two sinusoids. The vertical ticks on the time axis mark the timing of zero crossings in the uncontaminated signal. $B$, The fish's own EOD is generated internally, leading to current flow perpendicular to the skin surface at every point (black arrows), whereas the neighbor's EOD is generated externally, leading to current flow through the fish in a single direction (gray arrows). Because the electroreceptors are sensitive to current flow that is oriented perpendicular to the body surface, the fish's EOD will be more strongly contaminated at body surface A compared with B. C, Modulations in amplitude and phase at body surfaces $A$ and $B$ for opposite signs of Df. The vertical lines mark zero crossings. $D$, Amplitude at body surface $A$ and differential phase of body surface A relative to $B$ for opposite signs of Df. The temporal relationship between amplitude and differential phase is reversed when switching the sign of Df, which results in a different direction of rotation in a Lissajous graph. Adv., Advance; Del., delay.

neurons project to the torus semicircularis, where the two pathways converge onto combination-sensitive neurons, many of which are selective for the sign of Df (Kawasaki and Guo, 2002). This sign selectivity (SS) must result from nonlinear interactions between AM- and DPM-sensitive inputs, such that the response is dependent on the relative timing of synaptic inputs from the two pathways. In the current study, we characterized the response properties of single units to AM and PM to determine how responses to the two features interact to confer sign selectivity. This required a means of presenting AM and PM independently, which was achieved through the use of a "phase chamber," which divides the fish's body into electrically isolated head and trunk compartments (Heiligenberg and Bastian, 1980; Kawasaki, 1993).

\section{Materials and Methods}

Animals. A total of 21 Gymnarchus niloticus (10-25 cm in total length) were used. They were collected in West Africa at lengths of 5-6 cm, imported, and raised to the experimental size in our laboratory. Both sexes were used. Environmental conditions in the holding tanks were identical to those described previously (Kawasaki, 1994). After anesthesia with tricaine methanesulfonate (MS-222; 1:10,000; Sigma, St. Louis, $\mathrm{MO})$, we immobilized fish with an intramuscular injection of flaxedil (gallamine triethiodide; $8-150 \mu \mathrm{l}$ of a $0.1 \%$ solution; Sigma), which greatly attenuated EOD amplitude. Activity of the EOD pacemaker command signal was recorded from the tail to monitor the fish's condition throughout the experiments.

Fish were placed inside a Plexiglas chamber, gently held with a sponge- lined clamp, and submerged in water except for a small area along the dorsal surface of the head. Oxygen-saturated water was provided to the gills with a tube inserted in the mouth. After local application of Xylocaine (2\%; Barber Veterinary Supply, Richmond, VA), a small hole was drilled in the skull above the midbrain. These procedures are in accordance with guidelines established by the National Institutes of Health and were approved by the University of Virginia Institutional Animal Care and Use Committee.

Phase chamber electrosensory stimulation. After preparing the brain for recording, we electrically isolated the head and trunk into separate chambers by sliding Plexiglas doors against the fish's body, at a level just caudal to the posterior end of the skull, and sealed the gaps with a mixture of Vaseline and mineral oil (Fig. 2A). Stimuli to the head were provided through an anodal electrode placed in the mouth and two cathodal electrodes placed on either side of the fish's head. Stimuli to the trunk were provided through an anodal pin electrode placed in the dorsal musculature and two cathodal electrodes placed on either side of the fish's trunk. This allowed us to independently manipulate AM and PM in both the head and trunk compartments (Fig. $2 A)$. This experimental configuration can successfully elicit JARs as it approximates a natural situation in which a neighbor's electric field is longitudinal to the test fish (Kawasaki, 1993).

Sinusoidal electric stimuli, at a frequency within $20 \mathrm{~Hz}$ of the fish's EOD frequency before the experiment, were delivered to both chambers using homemade isolators with field effect transistors adjusted to a stimulus amplitude of $1-2 \mathrm{mV} / \mathrm{cm}$ as measured near the skin surface at the head. Each compartment in the phase chamber received a single sinusoidal signal, in which the carrier signal and any stimulus modulations were numerically generated by custom-made software for Matlab 6.5 (MathWorks, Natick, MA), which controlled a digital-toanalog board using a sampling rate of $20 \mathrm{kHz}$ (model DA3-4; TuckerDavis Technologies, Gainesville, FL). AM and PM could be generated in each chamber singly or could be combined using any temporal relationship, including those of a Df $>0$ stimulus ( $\mathrm{PM}$ start angle, $90^{\circ}$ relative to $\mathrm{AM}$ ) and $\mathrm{Df}<0$ stimulus (PM start angle, $270^{\circ}$ relative to AM). The depth of AM was set at $20 \%$ of the signal amplitude, whereas the depth of PM was set at $20^{\circ}$ relative to the carrier cycle for all stimuli.

Electrophysiology. Extracellular glass microelectrodes were pulled using a Flaming/Brown micropipette puller (model P-97; Sutter Instruments, Novato, CA) and broken to a tip diameter of 5-12 $\mu \mathrm{m}$. Electrodes were filled with a solution of either $3 \mathrm{M} \mathrm{NaCl}$ or $3 \mathrm{M} \mathrm{KCl}$ or were filled with Woods metal and electroplated with gold and platinum following Dowben and Rose (1953). Extracellular single-unit activity was amplified $1000 \times$ on a custom-made differential AC amplifier using a chlorided silver electrode placed on the brain surface as a reference. The resulting signal was sent to a Schmitt Trigger with an output to an event timer that recorded spike times using a clock rate of $1 \mathrm{MHz}$ (model ET1; Tucker-Davis Technologies). Spike times were saved using custom-made software for Matlab 6.5 (MathWorks).

The torus semicircularis is a large midbrain structure located immediately below the cerebellum and the optic tectum (Bass and Hopkins, 1982). The anterodorsal surface of the torus semicircularis could be visualized by gently pushing the optic tectum aside for electrode placement. One recording site was marked with Alcian Blue for later histological verification of the recording site (Kawasaki et al., 1988). Single electrosensory units in the torus were isolated by slowly advancing and retracting the electrode with a microelectrode drive (Inchworm 6000; Burleigh Instruments, Fishers, NY) and listening on an audio monitor for spikes that were modulated with each cycle of a $2 \mathrm{~Hz}$ search stimulus that alternated between head Df $<0$, head Df $>0$, trunk $\mathrm{Df}<0$, and trunk Df $>0$. After isolating a unit, a total of 10 different stimulus modulations were presented at modulation rates of 1, 2, or $4 \mathrm{~Hz}$ : AM, PM, Df $>0$, and $\mathrm{Df}<0$ in one compartment with no modulation in the other compartment (a total of eight stimuli; four for modulation in the head compartment and four for modulation in the trunk compartment); AM in both compartments (joint AM); and $\mathrm{PM}$ in both compartments (joint PM). Data were also recorded during no modulation in either compartment to obtain a baseline mea- 
A

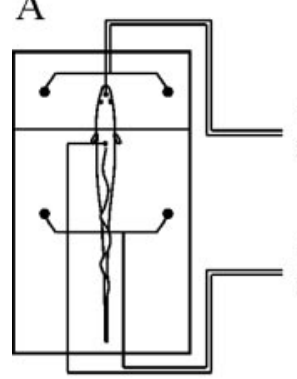

head
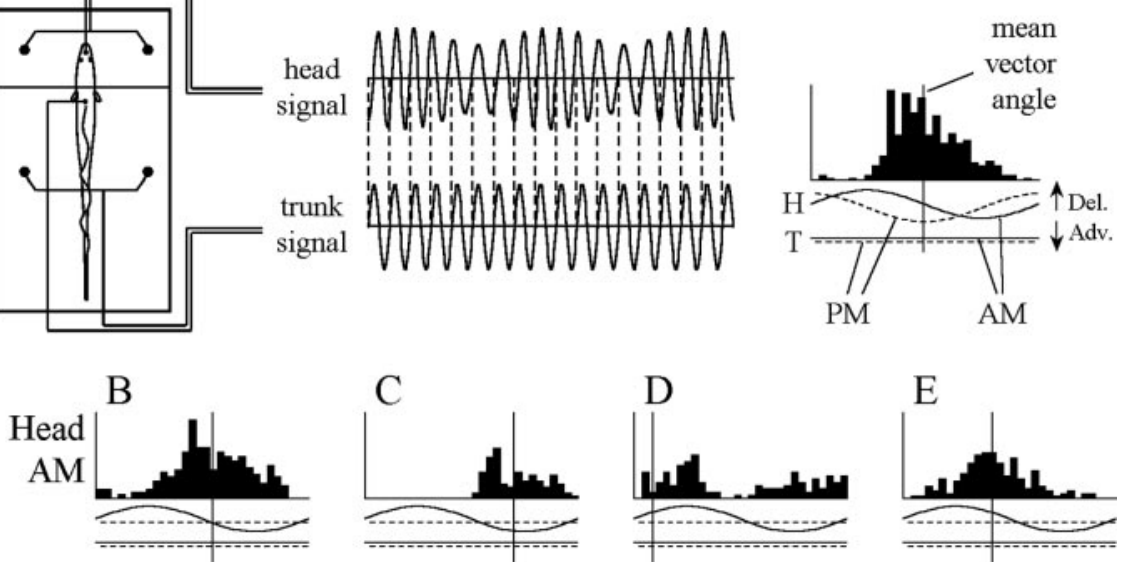

$\mathrm{C}$

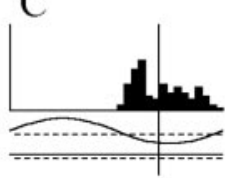

$\mathrm{D}$

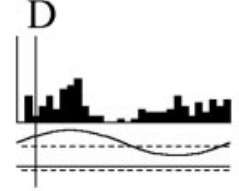

$\mathrm{E}$
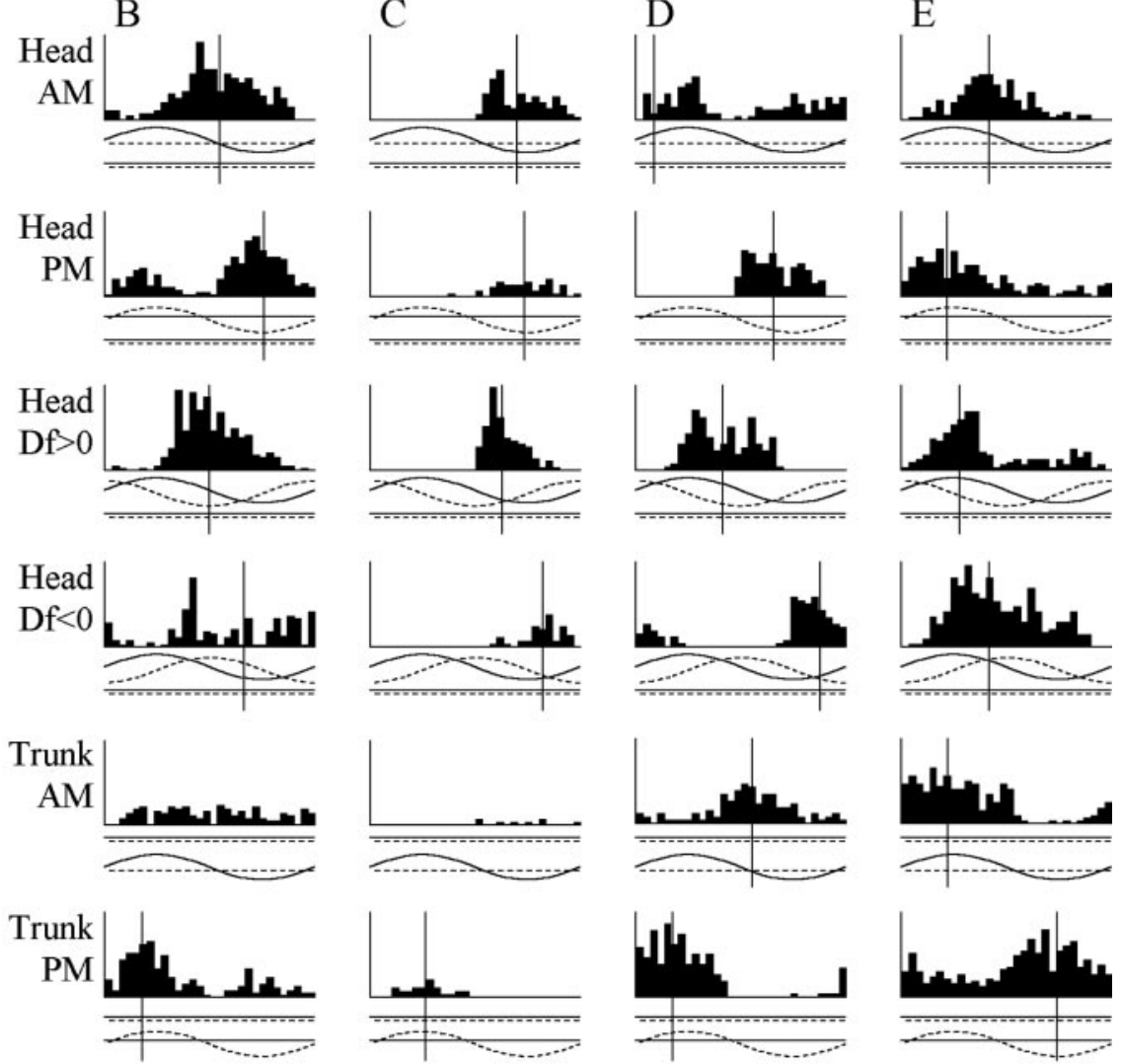

Trunk

Df $>0$
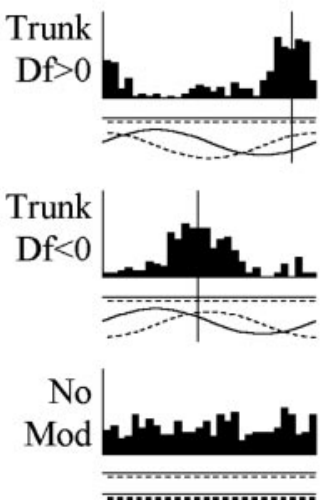
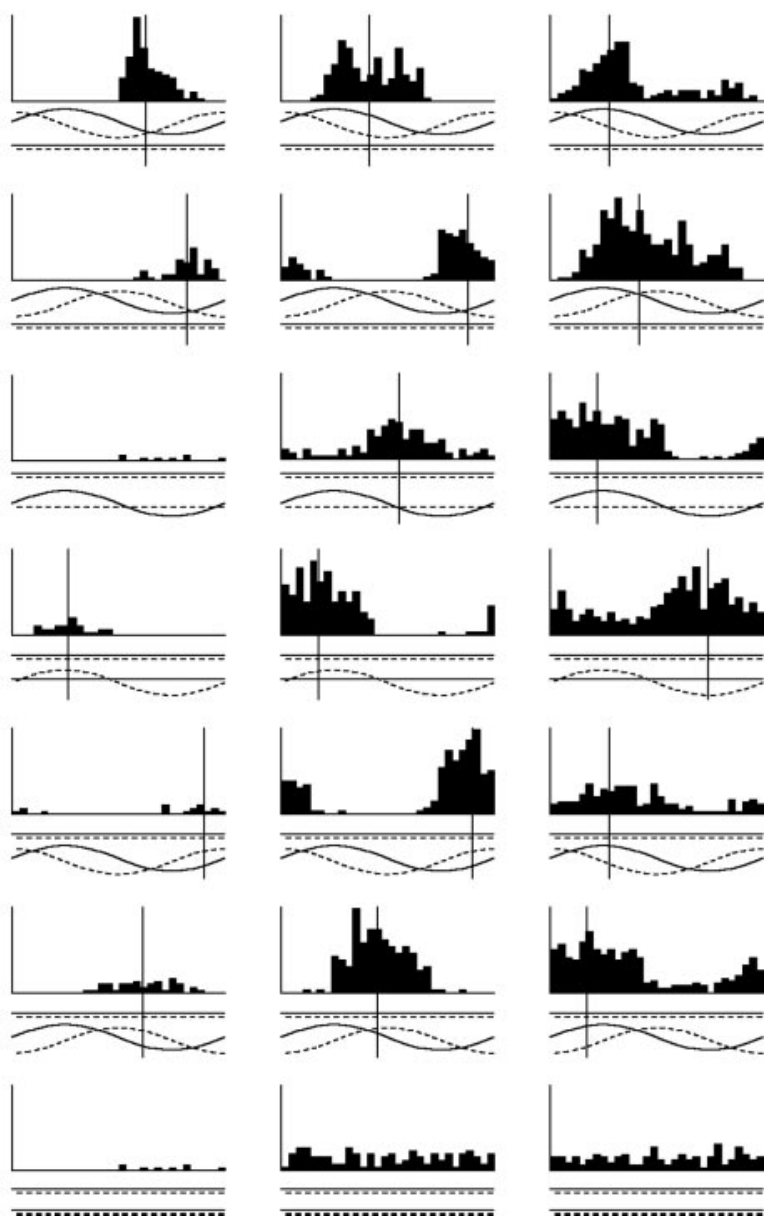

Figure2. A,Thephasechamberelectricallyisolates theheadfrom thetrunk, allowing onetoindependentlymanipulateAMandPM. Peristimulus timehistograms ofsingle-unitactivityare constructed in relationtothemodulation cycle. AM isshownasasolid line, and PMisshown as adashedline with delays indicated by positive values and advances by negativevalues. A vertical line is induded for all histograms with a significant $V S_{D f}$ at a value equal to the mean vector angle of the response. $B-D$, Responses of four different combination-sensitive units to $\mathrm{AM}, \mathrm{PM}, \mathrm{Df}>0$, and $\mathrm{Df}<0$ in both the head and trunk compartments as well as no modulation. The units in $B$ and $C$ were sensitive to head AM and DPM. Both gave an I/advance-type responsein the head and were $D f>0$ selective $\left(B, t_{82}=5.11, p<0.0001 ; C, t_{40}=4.05, p<0.001\right)$. The unitsin $D$ and Eweresensitiveto DAMand DPM. The unit in $D$ gave an $E /$ advance-type response in the head and an $1 /$ delay-type response in the trunk and was nonselective in both the head $\left(t_{40}=1.99 ; p>0.05\right)$ and thetrunk $\left(t_{40}=0.48 ; p>0.63\right)$. The unitinEgaveanl/delay-typeresponsein theheadandanE/advance-typeresponse in the trunk and was $\mathrm{Df}<0$ selective in both compartments (head, $t_{41}=8.65, p<0.000000001$; trunk, $t_{42}=4.34, p<0.001$ ). Vertical scale: $B$, 54 spikes/sec; $C, 45$ spikes/sec; D, 40.5 spikes/sec; $E$, 36 spikes/sec. Adv., Advance; Del., delay; No Mod, no modulation.

sure of activity. Clean single-unit isolation was maintained for the entire duration of the standard set of stimuli, which lasted for $\sim 5$ min. For some units that were held long enough, the standard set of 11 stimuli was presented multiple times using different modulation rates.

In one set of experiments designed to assess the importance of the relative timing of AM- and PM-induced activity on response linearity, units defined as sign selective (see below) were presented with stimuli in which the start angle of PM relative to AM was shifted from 0 to $340^{\circ}$ in $20^{\circ}$ steps. In a second set of experiments designed to determine the degree of orientation ambiguity in singleunit responses, we used stimuli in which both chambers were presented with the same sign of Df, but the depth of modulation in one chamber was set at $30 \%$ that of the other. Thus, the following four stimuli were presented: Df $>0$ (head $>$ trunk), Df $<0$ (head $>$ trunk), Df $>0$ (head $<$ trunk), and Df $<0$ (head $<$ trunk). These stimuli provide electrosensory input approximating that of natural jamming stimuli in different orientations in which the degree of contamination from a neighbor's EOD varies across the body surface (Kawasaki, 1993).

Data analysis. Spike histograms were constructed relative to the stimulus modulation cycle (Fig. 2A), and mean spike rates were determined for each cycle. A unit was defined as sign selective in a given chamber if the mean spike rates in response to opposite signs of Df were significantly different, measured using a $t$ test (two-tailed $\alpha=0.05$ ) as described by Kawasaki and Guo (2002).

We also determined the vector strength of synchronization (VS), which measures the degree of phase locking to the stimulus and varies from 0 (no synchronization) to 1 (complete synchronization) (Goldberg and Brown, 1969). This value was computed relative to the carrier signal $\left(\mathrm{VS}_{\text {car }}\right)$ as well as to the modulation cycle $\left(\mathrm{VS}_{\mathrm{Df}}\right)$. Units were defined as AM and/or PM sensitive if a significant $\mathrm{VS}_{\mathrm{Df}}$ occurred in response to these stimuli, as determined by a Rayleigh $z$ test with $\alpha=0.05$ (Batschelet, 1981). The relative timing of responses to each stimulus was determined as the mean vector angle of spike times in relation to the stimulus modulation cycle (Batschelet, 1981), with $0^{\circ}$ corresponding to the start of the cycle, $180^{\circ}$ corresponding to the center of the cycle, and $360^{\circ}$ corresponding to the end of the cycle (Fig. 2A). AMsensitive units were defined as "E-type" if they responded during amplitude increases and "I-type" if they responded during amplitude decreases. PM-sensitive units were defined as "delay-type" if they responded to phase delays and "advance-type" if they responded to phase advances. For Df $<0$ and Df $>0$ responses, the absolute value of the angular distance between the AM response angle and the PM response angle was used to determine the relative timing of responses to the two stimulus components. This value 
varied from $0^{\circ}$ (response angles aligned) to $180^{\circ}$ (response angles completely out of phase).

The linearity of responses to Df $>0$ and Df $<0$ was assessed in the following way. Response histograms to AM and PM stimulation were constructed as the number of spikes per modulation cycle, using a total of 48 bins. To normalize these responses as a change in baseline firing rate, we subtracted from each bin the average number of spikes per bin in response to no stimulus modulation. The PM response histogram was then shifted by $90^{\circ}$ for Df $>0$ and $270^{\circ}$ for Df $<0$ to align the expected response to $\mathrm{PM}$ with the combination stimulus. Finally, the resulting $\mathrm{AM}$ and PM histograms were both added to the baseline firing rate to create an expected linear combination of responses to each component. In the few cases that this resulted in negative expected firing rates, expected values were changed to 0 , because firing rates cannot be negative. To determine whether observed responses deviated significantly from linearity, the observed spike rates from each modulation cycle were tested against the expected spike rate derived from the expected linear response histogram using a single sample $t$ test (two-tailed; $\alpha=0.01$ ). Observed spike rates that were significantly lower than expected were classified as "suppression" responses, observed spike rates that were significantly greater than expected were classified as "facilitation" responses, and observed spike rates that were not significantly different from expected were classified as "linear" responses.

For the experiments testing orientation ambiguity, the mean spike rate was determined, and the sign selectivity (SS) of each unit was assessed for both head $>$ trunk stimuli and head $<$ trunk stimuli, computed as SS $=\left(\mathrm{spk}_{\mathrm{Df}>0}-\mathrm{spk}_{\mathrm{Df}<0}\right) /\left(\mathrm{spk}_{\mathrm{Df}>0}+\mathrm{spk}_{\mathrm{Df}<0}\right)$ where $\mathrm{spk}_{\mathrm{Df}>0}$ and $\mathrm{spk}_{\mathrm{Df}<0}$ are the mean spike rates for $\mathrm{Df}>0$ and $\mathrm{Df}<0$, respectively (Kawasaki and Guo, 2002). To measure the difference in SS for head $>$ trunk and head $<$ trunk stimuli, we developed an orientation-ambiguity index (OAI) that varied from 0 (identical SS) to 1 (opposite sign of SS), calculated as OAI $=0.5-\left[0.5^{\star}\left(\mathrm{SS}_{\min } /\right.\right.$ $\left.\mathrm{SS}_{\max }\right)$ ], where $\mathrm{SS}_{\min }$ and $\mathrm{SS}_{\max }$ are the smaller and larger of the two SS values, respectively.

All statistical procedures were done using Statistica 6.1 (StatSoft, Tulsa, OK) or custom-made software for Matlab 6.5.

\section{Results}

We recorded from a total of 61 tuberous electrosensory toral units. Of these, 11 responded solely to $\mathrm{AM}$, and 50 responded to both AM and PM (combination sensitive). The AM-sensitive units had a significantly lower degree of phase locking to the carrier signal $\left(\mathrm{VS}_{\mathrm{car}}\right)$ than the combination-sensitive units (mean \pm SEM, $0.13 \pm 0.02$ and $0.31 \pm 0.03$, respectively; MannWhitney $U$ Test, $z=2.00 ; p<0.05$ ).

\section{Sign selectivity of combination-sensitive units}

Responses of four representative combination-sensitive units are shown in Figure 2. All 50 combination-sensitive units showed DPM-sensitive responses, with 24 responding to phase advances in the head and phase delays in the trunk (Fig. $2 B-D$ ) and 26 responding to phase delays in the head and phase advances in the trunk (Fig. 2 E). In response to AM, 29 of these units responded in only one compartment (11 E-type, 18 I-type) (Fig. $2 B, C$ ). One unit gave an I-type response in both compartments, and one unit gave an E-type response in both compartments, indicating that the receptive fields of these units spanned the border between chambers. Nineteen of these units responded differentially to AM in each chamber, with 12 showing an E-type response in the head and I-type response in the trunk (Fig. 2D) and 7 showing an I-type response in the head and E-type response in the trunk (Fig. $2 E)$. These units were therefore classified as differential AM sensitive (DAM sensitive).

Of the 50 combination-sensitive units, 37 were sign selective for at least one of the modulation rates tested. Df $<0$ selective responses typically occurred when the mean vector angles of the AM and PM responses were more closely aligned for Df $<0$ (mean angular distance \pm SEM, $58.53 \pm 7.56^{\circ}$ ) than for $D f>0\left(121.46 \pm 7.57^{\circ}\right)$, a difference that was significant (Wilcoxon matched pairs test, $z_{22}=3.23$; $p<0.01$ ). In contrast, Df $>0$-selective responses typically occurred when the mean vector angles of the AM and $\mathrm{PM}$ responses were more closely aligned for Df $>0\left(72.12 \pm 6.55^{\circ}\right)$ than for Df $<0$ $\left(107.88 \pm 6.55^{\circ}\right)$, which was also a significant difference $\left(z_{38}=\right.$ $2.50 ; p<0.05)$. However, nonselective responses typically occurred when the mean vector angles of the AM and PM responses were approximately equally aligned for both $\mathrm{Df}<0$ and Df $>0\left(z_{51}=1.08 ; p>0.25\right)$.

For example, the units in Figure 2, $B$ and $C$, both show an I/advance-type response in the head; these two responses are more closely aligned for $\mathrm{Df}>0$ than $\mathrm{Df}<0$, and these units are therefore sign selective for Df $>0$ in the head. The unit in Figure $2 E$ gives an I/delay-type response in the head and an 

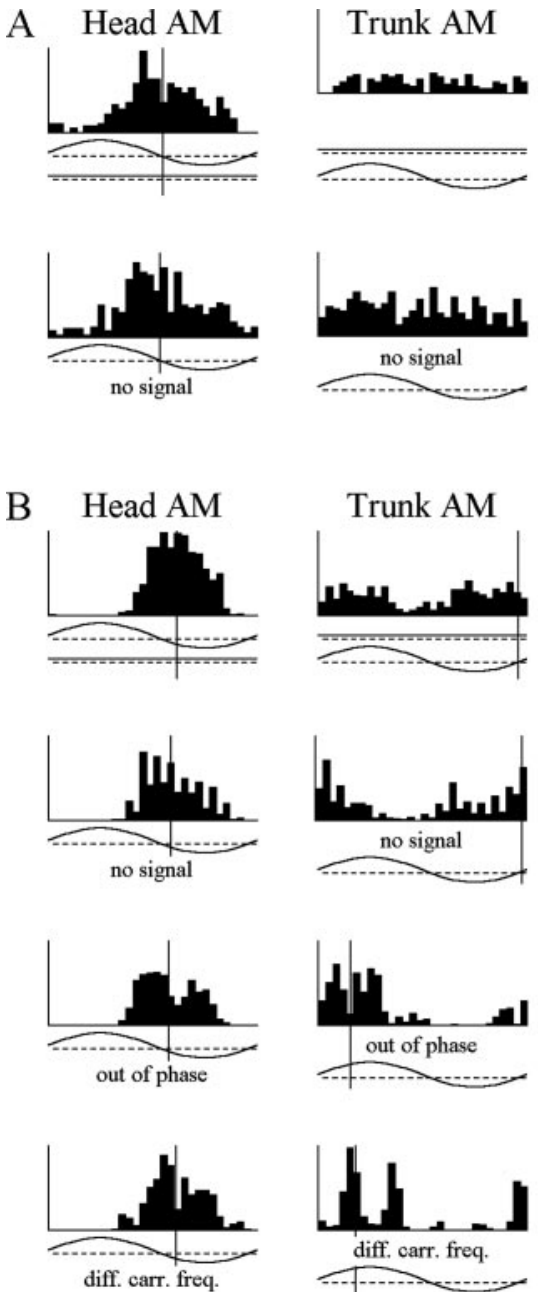
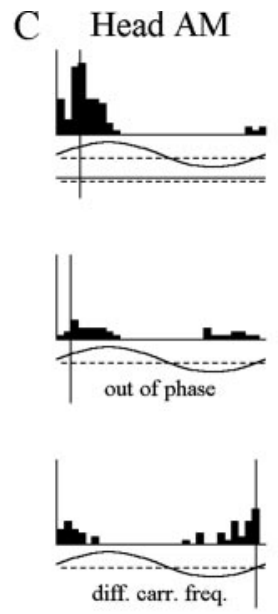

D
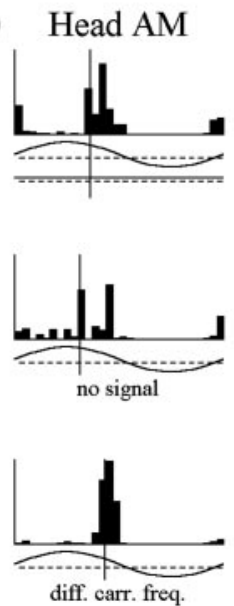

Figure 4. Verification of DAM sensitivity. $A$, This AM-sensitive unit responded to head AM, regardless of whether there was any signal in the trunk compartment, but gave no response to trunk $A M$. Vertical scale, 51 spikes $/ \mathrm{sec}$. The units in $B-D$ were all DAM sensitive and responded to AM in both compartments under a variety of different stimulus conditions, including no signal in the other compartment, signals of different carrier frequencies (diff. carr. freq.) in the two compartments, and signals in the two compartments with $180^{\circ}$ phase differences. Vertical scale: $B, 132$ spikes/sec; $C, 30$ spikes/sec; $D, 96$ spikes $/ \mathrm{sec}$.

Table 1. Linearity of responses in relation to the sign selectivity of combinationsensitive units

\begin{tabular}{lccc}
\hline & \multicolumn{3}{c}{ Response to Df $<0$} \\
\cline { 2 - 4 } Response to Df $>0$ & Suppression & Linear & Facilitation \\
\hline Nonselective responses & & & \\
$\quad$ Suppression & 17 & 15 & 5 \\
$\quad$ Linear & 5 & 4 & 3 \\
$\quad$ Facilitation & 0 & & \\
Df $<$-selective responses & & 7 & 2 \\
$\quad$ Suppression & 0 & 1 & 3 \\
Linear & 0 & 0 & 1 \\
$\quad$ Facilitation & & & 0 \\
Df $>$ 0-selective responses & 11 & 0 & 0 \\
$\quad$ Suppression & 8 & 3 & 0 \\
Linear & 5 & 11 & \\
Facilitation & & &
\end{tabular}

Units are represented more than once if they were stimulated with more than one modulation rate or if they were DAM sensitive, in which case stimulation of both the head and trunk chamber are included.

E/advance-type response in the trunk. These two responses are more closely aligned for Df $<0$ than Df $>0$, and this unit is therefore sign selective for Df $<0$ in both chambers. In contrast, the unit in Figure $2 \mathrm{D}$ does not show a sign-selective
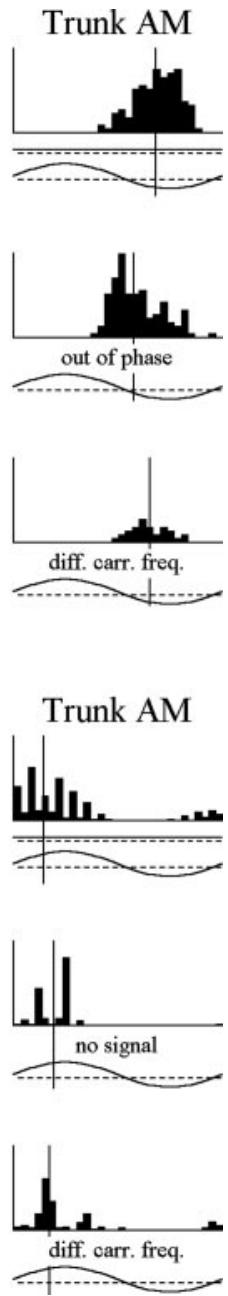

response. Although it responds to $\mathrm{AM}$ and PM in both chambers, the mean vector angles are approximately equally aligned for both signs of Df.

\section{Differential AM sensitivity}

Although AM-sensitive units showed identical responses to compartmental and joint AM (Wilcoxon matched pairs test, $z=0.30 ; p>0.75$ ) (Fig. 3A), DAMsensitive units had a significantly lower spike rate in response to joint $\mathrm{AM}(z=$ 3.26; $p<0.01$ ) (Fig. 3B-E), which generally appeared as a reduction in the response to either head AM (Fig. 3E) or trunk AM (Fig. 3B-D). Thus, differential AM sensitivity may arise from dual E-type and I-type inputs from separate parts of the body surface. However, DPM-sensitive units in the electrosensory lateral line lobe sometimes respond to AM, because the time-coding afferents that phase lock to electrosensory input sometimes show an amplitudedependent latency shift (Kawasaki and Guo, 1996). Thus, DAM-sensitive responses could be artifacts caused by a DPM-sensitive unit responding to AMinduced shifts in spike times.

Several observations suggest this is not the case. First, not every DPMsensitive unit was also DAM sensitive (Fig. 3A). Second, we recorded from one unit that was DAM sensitive but showed no response to PM in either compartment (Fig. 3B). Last, if DAM sensitivity were a byproduct of DPM sensitivity, then every DAM-DPM-sensitive unit would give either an E/advance or I/delay response, because spike latencies decrease with increasing amplitude and increase with decreasing amplitude. However, this was not always the case, because some DAM-DPM-sensitive units gave I/advance or E/delay responses (Fig. 3C). Indeed, there was no significant association between DAM and DPM sensitivity (Fisher's exact test, $p>0.6$ ).

To experimentally verify whether DAM-sensitive responses were an artifact of DPM sensitivity, eight DAM-sensitive units were tested with at least one of the following three stimuli: AM in one compartment and no carrier signal in the other compartment, $\mathrm{AM}$ in one compartment and an unmodulated signal with stationary phase offset by $180^{\circ}$ in the other compartment, and AM in one compartment and an unmodulated signal with a different carrier frequency in the other compartment. These three types of stimuli eliminate phase information as a potential contributing factor to DAM sensitivity. All eight DAM-sensitive units continued to respond to AM in each compartment for all three stimuli (Fig. 4), verifying that DAM sensitivity results from AM-sensitive inputs.

\section{Nonlinear interactions between AM and PM}

Combination-sensitive units exhibited remarkable diversity in the linearity of their response patterns. Nonselective responses were typically characterized by identical interactions between 
$\mathrm{AM}$ and $\mathrm{PM}$ responses to both signs of Df, consisting mostly of linear-linear and suppression-suppression responses (Table 1). In contrast, sign-selective responses were far more divergent, with greater frequencies of linear-facilitation, linear-suppression, and facilitation-suppression responses (Table 1). When comparing nonselective, Df $<0$-selective, and Df $>0$ selective responses, the difference in the distribution of interaction types was highly significant (partial association, $X_{2}{ }^{2}=10.5 ; p<$ $0.01)$. Several examples of linear and nonlinear interactions are shown in Figure 5.

Among sign-selective responses in which the response to one sign of Df was linear and the response to the other sign of Df was nonlinear, facilitation tended to occur when the AM and PM response angles were aligned (mean angular distance \pm SEM, $68.79 \pm 10.83^{\circ}$ ), whereas suppression tended to occur when the $\mathrm{AM}$ and $\mathrm{PM}$ response angles were offset $\left(105.36 \pm 9.42^{\circ}\right)$, a difference that was significant (Mann-Whitney $U$ test, $z=$ 2.40; $p<0.05$ ). For example, the unit shown in Figure $5 B$ gave a strong I-type response to head AM and a moderate delay-type response to head PM and was characterized as Df $>0$ selective. Facilitation between the AM and PM responses occurred in response to Df $>0$, when the response angles were closely aligned, but these responses summated linearly in response to Df $<0$ when the response angles were offset. The units shown in Figure 5, $C$ and $D$, gave signselective responses resulting from suppression when the AM and PM response angles were offset and linear summation when they were aligned. The response patterns of the unit in Figure $5 D$ are especially dramatic, because the response to PM alone is nearly imperceptible, but it dramatically suppresses the AM response when their angles are offset. The unit shown in Figure $5 E$ gives an example of a unit that showed facilitation in response to Df $>0$ when the response angles were aligned and suppression in response to Df $<0$ when the response angles were offset.

We tested the responses of three Df $<0$-selective units and six Df $>0$-selective units to stimuli in which the PM start angle relative to $\mathrm{AM}$ was set at values ranging from 0 to $340^{\circ}$. The spike rates of these units were highly dependent on this angular value, with $\mathrm{Df}<0$-selective units showing peak responses at values similar to a natural Df $<0$ stimulus $\left(\sim 270^{\circ}\right)$ (Fig. $6 A$ ) and $\mathrm{Df}>0$-selective units showing peak responses at values similar to a natural Df $>0$ stimulus $\left(\sim 90^{\circ}\right)$ (Fig. $\left.6 \mathrm{~B}\right)$. To test the significance of the relative timing of responses to AM and PM, PM start angles were converted into angular distances between AM and PM mean response vector angles across all units tested with these stimuli. Responses to AM and PM combinations were highly dependent on the relative timing of AM and PM response vectors, with the greatest spike rates occurring when these two angles were closely aligned (Fig. 6C).

\section{Silent PM responses}

Some of the 11 units designated as solely AM sensitive (no significant response to PM presented alone) gave sign-selective responses when AM and PM were combined (Fig. 7). In some cases, this sign selectivity resulted from an increase in spike rate compared with AM for one sign of Df but not the other. For example, the unit in Figure $7 A$ responded with a significantly greater spike rate to $\mathrm{Df}>0$ than to $\mathrm{AM}\left(t_{82}=5.67 ; p<0.000001\right)$, but there was no significant difference in responses to $\mathrm{Df}<0$ and $\mathrm{AM}\left(t_{82}=\right.$ $0.68 ; p>0.45)$. Similarly, the unit in Figure $7 B$ gave similar spike rates in response to $\mathrm{Df}>0$ and $\mathrm{AM}\left(t_{59}=0.36 ; p>0.72\right)$ but a greater spike rate in response to Df $<0$ than to $A M$, although this difference was not quite significant $\left(t_{63}=1.77 ; p<0.09\right)$. In other cases, this sign selectivity resulted from a decrease in spike rate compared with $\mathrm{AM}$ for one sign of Df but not the other. For example, the unit in Figure $7 C$ responded with a significantly lower spike rate to $\mathrm{Df}<0$ than to $\mathrm{AM}\left(t_{82}=3.15\right.$; $\left.p<0.01\right)$, but there was no significant difference in the responses to Df $>0$ and $\operatorname{AM}\left(t_{82}=1.19 ; p>0.23\right)$.

\section{Orientation ambiguity}

To determine the degree of orientation ambiguity in five AMDPM-sensitive units and four DAM-DPM-sensitive units, both 

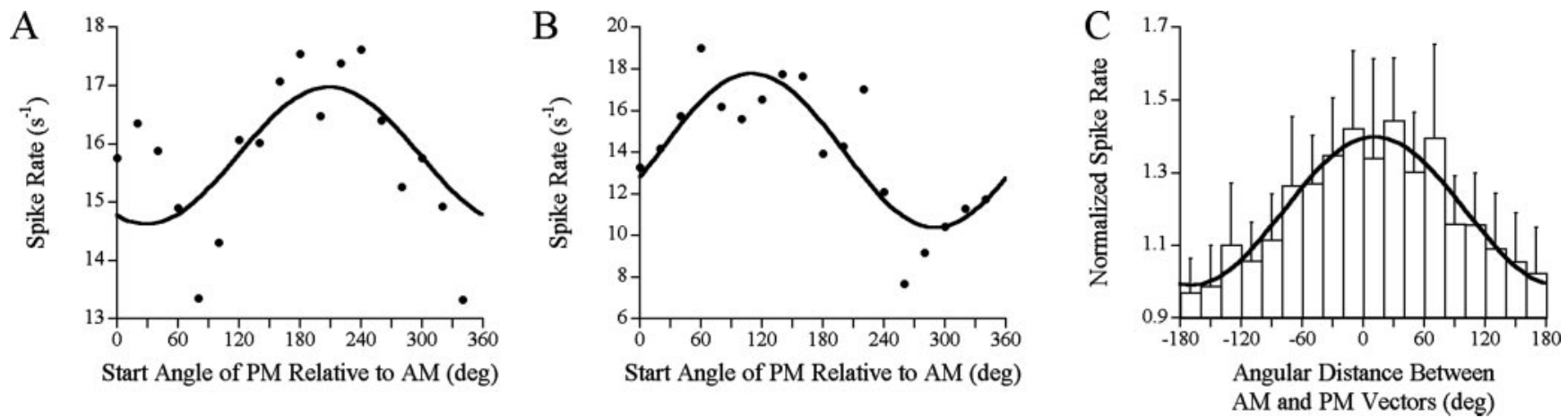

Figure 6. Responses to various start angles of PM relative to $A M$ in sign-selective units. $A$, This unit was $D f<0$ selective and responded most strongly when $P M$ was offset by values near that of a natural $\mathrm{Df}<0$ stimulus $\left(270^{\circ}\right)$. The fitted function is of the form $y=M+A \cos \left(t-t_{0}\right)\left(r^{2}=0.44 ; p<0.01\right)$. B, This unit was $\mathrm{Df}>0$ selective and responded most strongly when PM was offset by values near that of a natural $\mathrm{Df}>0$ stimulus $\left(90^{\circ}\right)$. The fitted function is of the same form in $A\left(r^{2}=0.72 ; p<0.00001\right)$. C, Ten responses from nine units to the same start angles shown in $A$ and $B$, shifted to show the relative distance between the AM and PM mean vector angles and then binned into $20^{\circ}$ segments. Spike rate is normalized to the spike rate in response to $\mathrm{AM}$ alone (mean \pm SEM), with a fitted function of the same form in $A$ and $B\left(r^{2}=0.91 ; p<0.0000001\right)$.
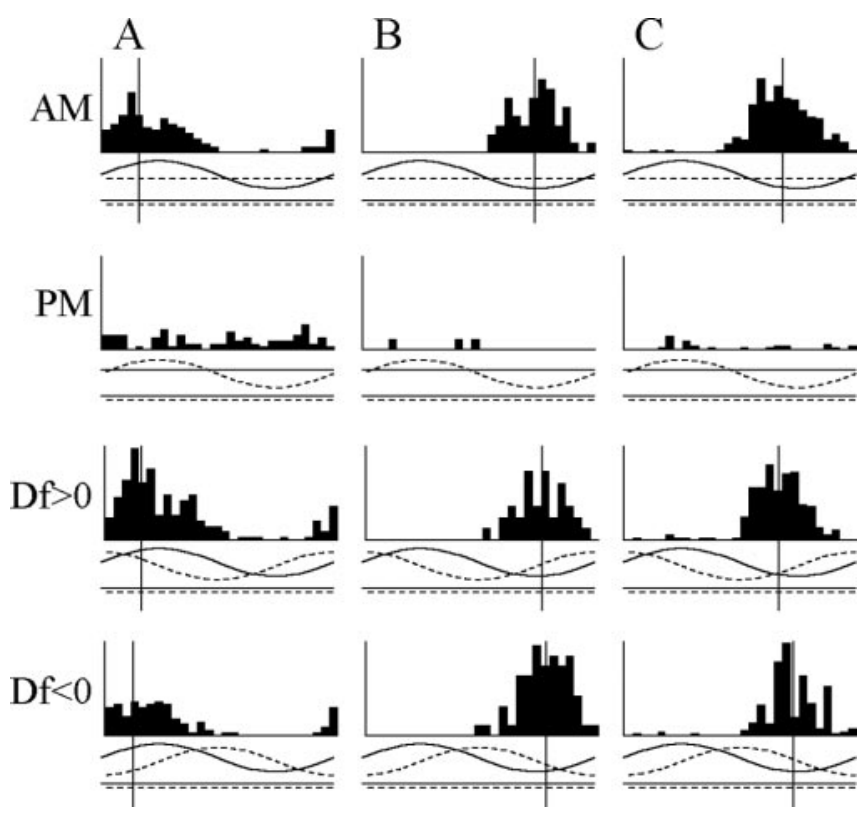

Figure 7. Sign selectivity in three units that gave no observable response to $\mathrm{PM}$ presented alone. $A$, Df $>0$-selective unit $\left(t_{82}=6.80 ; p<0.00000001\right)$. Vertical scale, 46.2 spikes $/ \mathrm{sec}$. $B$, Df $<0$-selective unit $\left(t_{56}=2.24 ; p<0.05\right)$. Vertical scale, 9 spikes $/$ sec. $C, D f>0$-selective unit $\left(t_{82}=2.10 ; p<0.05\right)$. Vertical scale, 77.4 spikes $/$ sec.

chambers were stimulated with identical signs of Df, but the depth of modulation in one chamber was set at $30 \%$ that of the other (Kawasaki, 1993). When the relative modulation strengths of stimulation in the two chambers were reversed, the SS reversed for AM-DPM-sensitive units, whereas it remained unchanged for DAM-DPM-sensitive units (Fig. 8). As a result, the orientation ambiguity index of AM-DPM-sensitive responses (mean \pm SEM, $0.791 \pm 0.048)$ was greater than that of DAM-DPMsensitive responses $(0.359 \pm 0.059)$, a difference that was significant (Mann-Whitney $U$ test, $z=2.74 ; p<0.01$ ).

\section{Discussion}

We found that many combination-sensitive electrosensory neurons in the torus of Gymnarchus showed sign-selective responses that were established through nonlinear interactions between AM- and PM-sensitive inputs. These nonlinear inter- actions were related to the relative timing of synaptic input from the two pathways: facilitation in response to the preferred sign of Df occurred when the peak responses to AM and PM were aligned, whereas suppression in response to the nonpreferred sign of Df occurred when the peak responses to AM and PM were offset. By presenting stimuli with AM and PM offset to varying degrees, we directly demonstrated that the resulting postsynaptic activity is strongly dependent on the relative timing of synaptic responses to each stimulus feature.

The gymnotiform electric fish Eigenmannia also produces a JAR, but its electrosensory and electromotor systems have evolved independently from Gymnarchus (Bullock et al., 1975, 1983). As in Gymnarchus, separate AM- and PM-sensitive pathways converge in the torus, where sign-selective neurons with nonlinear responses to combinations of AM and PM have also been described (Heiligenberg and Rose, 1986). We discovered a novel type of electrosensory neuron in Gymnarchus, DAM-sensitive units that respond to differences in amplitude between two body surfaces. This DAM sensitivity appears to result from an E-type input from one part of the body surface and an I-type input from another. Their existence has been reported in the torus of Eigenmannia (Partridge et al., 1981), but they appear to be extremely rare and have therefore not been well characterized. In contrast, approximately one-third of the combination-sensitive units we recorded in Gymnarchus were DAM sensitive.

DAM-sensitive units may play a significant role in the JAR because they provide a solution to the problem of orientation ambiguity, which arises from the fact that AM is encoded in an absolute sense, but PM is encoded by determining the relative difference in phase between two body surfaces (Kawasaki, 1993). Considering the example shown in Figure 1, for the Df $>0$ stimulus, a neuron that compares the phase of area $\mathrm{A}$ relative to area $\mathrm{B}$ receives sensory input signaling $\mathrm{Df}>0$, whereas a neuron that compares the phase of area $B$ relative to area A receives sensory input signaling $\mathrm{Df}<0$. If the sign of Df remained the same but the orientation of the stimulus were to change, such that area B became more strongly contaminated than area $A$, then the sign of Df seen by these two neurons would flip. Indeed, changes in the orientation of free-field jamming stimuli often alter the sign selectivity of toral neurons in both Eigenmannia and Gymnarchus (Rose and Heiligenberg, 1986; Kawasaki and Guo, 2002). 


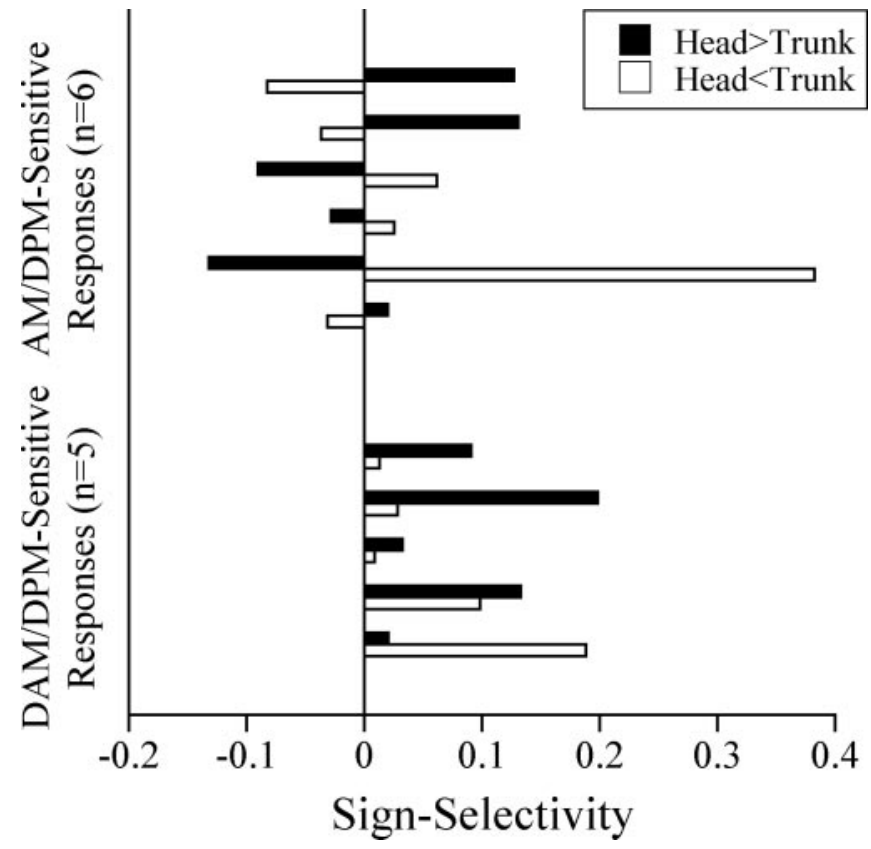

Figure 8. Orientation ambiguity in AM-DPM-sensitive units and DAM-DPM-sensitive units. Sign selectivity of responses from five AM-DPM-sensitive units and four DAM-DPMsensitive units (1 unit from each group was stimulated with 2 different modulation rates) in response to joint stimulation of the head and trunk with identical signs of Df but with the modulation depth of one chamber set at $30 \%$ of the other.

Theoretically, the sign selectivity of a neuron receiving DAM and DPM inputs should be less affected by changes in stimulus orientation than a neuron receiving AM and DPM inputs. Just as the difference in phase between two body surfaces will be reversed for different stimulus orientations, so too will the difference in amplitude, and the temporal relationship between DAM and DPM will therefore remain unchanged. Indeed, we showed that units receiving AM and DPM inputs showed greater orientation ambiguity than units receiving DAM and DPM inputs. In fact, the latter always gave consistent sign-selective responses regardless of which chamber was more strongly stimulated, whereas the former always gave inconsistent sign-selective responses.

The relative role of DAM-DPM-sensitive units and AMDPM-sensitive units in the JAR of Gymnarchus remains unclear. In Eigenmannia, the solution to orientation ambiguity appears to involve pooling the responses of sign-selective AMDPM-sensitive toral neurons, which together provide unambiguous information on the sign of Df (Heiligenberg and Rose, 1986). Thus, AM-DPM-sensitive units in Gymnarchus could still be involved in the JAR. In addition, DAM sensitivity could be important for other electrosensory processes. For example, the closely related mormyrid electric fish Gnathonemus petersii can use electrolocation to measure the distance to an object, an ability that appears to rely on, in part, measuring the slope of the electric field amplitude along the body surface (von der Emde et al., 1998). DAM-sensitive neurons could play an important role in electrolocation by measuring this slope as the difference in amplitude between different portions of the body surface.

The motor pathway controlling the JAR in Gymnarchus remains to be explored. In Eigenmannia, there are two additional processing steps between the torus and the medullary pacemaker nucleus (Pn) that controls EOD frequency (Heiligenberg, 1991). Sign-selective neurons in the torus project to a diencephalic structure termed the nucleus electrosensorius, in which there are two distinct subdivisions involved in the JAR, one with neurons selective to Df $<0$ and a second with neurons selective to Df $>0$ (Keller, 1988; Keller and Heiligenberg, 1989). The former projects to the central posterior-prepacemaker nucleus, which drives EOD frequency increases, whereas the latter projects to the sublemniscal prepacemaker nucleus, which mediates EOD frequency decreases (Keller et al., 1990; Metzner, 1993). It is likely that an additional sensory processing step after the torus is necessary in Gymnarchus as well. Although DAM-sensitive units consistently responded with a greater spike rate to one sign of Df over the other regardless of which chamber was more strongly stimulated, the lower spike rate in one chamber for the nonpreferred sign of Df was sometimes larger than the greater spike rate in the other chamber for the preferred sign. Thus, even in DAMsensitive units, spike rate does not, by itself, unambiguously encode the sign of Df.

Combination-sensitive neurons play a critical role in behavior because information about a specific stimulus parameter often holds biological relevance only when combined with information about other parameters. For instance, barn owls are able to locate sounds in two dimensions by combining interaural time differences with interaural intensity differences to determine azimuth and elevation, respectively (Moiseff, 1989). These two types of information are processed in parallel pathways that converge onto combination-sensitive neurons in the inferior colliculus, which forms a map of auditory space (Knudsen and Konishi, 1978; Moiseff and Konishi, 1983). Neurons in the auditory cortex of echolocating bats are sensitive to specific combinations of features in outgoing sound pulses and incoming echoes, which provides information about target characteristics such as range and relative velocity (Suga et al., 1978; Olsen and Suga, 1991a,b). The mating calls of certain frogs contain both low- and high-frequency components, and call recognition appears to be mediated by auditory neurons that respond selectively to the presence of both (Fuzessery and Feng, 1983). Other examples of combination-sensitive neurons include auditory neurons in songbirds, primates, and bats that respond selectively to the presence of multiple components in species-specific calls (Margoliash, 1983; Olsen and Rauschecker, 1992; Ohlemiller et al., 1994; Doupe, 1997) and individual face recognition neurons in the inferotemporal cortex of primates that respond selectively to the presence of multiple facial features (Perrett et al., 1982). It has even been suggested that combinationsensitive auditory neurons may be involved in spoken language comprehension in humans (Sussman et al., 1998).

Despite the importance of combination-sensitive neurons to sensory processing in vertebrates, it remains unclear how their unique physiological properties are established at the synaptic level. Responses to presentation of AM or PM alone could result from excitation occurring at the response peak, inhibition occurring out of phase with the response peak, or both. Facilitation in spike rates could result from a simple linear summation of postsynaptic potentials if the spike threshold were greater than the potential generated by one input alone but less than that generated by both inputs (Srinivasan and Bernard, 1976). NMDA receptors could drive supralinear summations of concurrent EPSPs (Mel, 1992, 1993). Neurons could multiply simultaneous inputs by computing 
the "log-exp transform" if postsynaptic potentials are logarithmically related to input spike rates, these potentials are linearly summated, and postsynaptic spike rate is an exponential function of membrane potential. Recent evidence suggests that this kind of operation is involved in visually guided collision avoidance in locusts (Gabbiani et al., 2002). Suppression is likely driven by inhibitory inputs. Inhibitory responses to one stimulus feature alone could be weak in neurons with low resting firing rates or with reversal potentials near the resting membrane potential but could strongly shunt excitatory responses to the other stimulus feature (Torre and Poggio, 1978). The reverse could also drive facilitation through disinhibition: the response to one stimulus feature could be a removal of inhibition that otherwise blocks the excitatory response to the other stimulus feature (Grzywacz and Poggio, 1990).

The JAR of weakly electric fish is an ideal model system for addressing these hypotheses. The behavior is highly robust and relies on just two stimulus parameters that may be easily and independently manipulated. Combination-sensitive electrosensory toral neurons in Gymnarchus have already been recorded from in vivo using the whole-cell intracellular technique (Kawasaki and Guo, 2002). Future experiments will combine independent manipulation of AM and PM using the phase chamber with whole-cell recordings. This will allow us to characterize the postsynaptic potentials involved and how they influence each other as well as describe the relationship between membrane potential and spike rate. Our findings should prove informative to the general problem of how nonlinear responses are established in combination-sensitive neurons.

\section{References}

Bass AH, Hopkins CD (1982) Comparative aspects of brain organization of an African wave electric fish, Gymnarchus niloticus. J Morphol 174:313-334.

Batschelet E (1981) Circular statistics in biology, pp 54-58. New York: Academic.

Bullock TH, Behrend K, Heiligenberg W (1975) Comparison of the jamming avoidance responses in gymnotoid and gymnarchid electric fish: a case of convergent evolution of behavior and its sensory basis. J Comp Physiol [A] 103:97-121.

Bullock TH, Bodznick DA, Northcutt RG (1983) The phylogenetic distribution of electroreception: evidence for convergent evolution of a primitive vertebrate sense modality. Brain Res Brain Res Rev 6:25-46.

Doupe AJ (1997) Song- and order-selective neurons in the songbird anterior forebrain and their emergence during vocal development. J Neurosci 17:1147-1167.

Dowben R, Rose J (1953) A metal-filled microelectrode. Science 118:22-24

Fuzessery ZM, Feng AS (1983) Mating call selectivity in the thalamus and midbrain of the leopard frog (Rana p. pipiens): single and multiunit analyses. J Comp Physiol [A] 150:333-344.

Gabbiani F, Krapp HG, Koch C, Laurent G (2002) Multiplicative computation in a visual neuron sensitive to looming. Nature 420:320-324.

Goldberg JM, Brown PB (1969) Response of binaural neurons of dog superior olivary complex to dichotic tonal stimuli: some physiological mechanisms of sound localization. J Neurophysiol 32:613-636.

Grzywacz NM, Poggio T (1990) Computation of motion by real neurons. In: An introduction to neural and electronic networks (Zornetzer SF, Davis JL, Lau C, eds), pp 379-403. Orlando: Academic.

Heiligenberg WF (1975) Electrolocation and jamming avoidance in the electric fish Gymnarchus niloticus (Gymnarchidae, Mormyriformes). J Comp Physiol [A] 103:55-67.

Heiligenberg WF (1991) Neural nets in electric fish. Cambridge: MIT.

Heiligenberg WF, Bastian J (1980) The control of Eigenmannia's pace- maker by distributed evaluation of electroreceptive afferences. J Comp Physiol [A] 136:113-133.

Heiligenberg WF, Rose G (1986) Gating of sensory information: joint computations of phase and amplitude data in the midbrain of the electric fish, Eigenmannia. J Comp Physiol [A] 159:311-324.

Kawasaki M (1993) Independently evolved jamming avoidance responses employ identical computational algorithms: a behavioral study of the African electric fish, Gymnarchus niloticus. J Comp Physiol [A] 173:9-22.

Kawasaki M (1994) The African wave-type electric fish, Gymnarchus niloticus, lacks corollary discharge mechanisms for electrosensory gating. J Comp Physiol [A] 174:133-144.

Kawasaki M, Guo Y-X (1996) Neuronal circuitry for comparison of timing in the electrosensory lateral line lobe of the African wave-type electric fish Gymnarchus niloticus. J Neurosci 16:380-391.

Kawasaki M, Guo Y-X (1998) Parallel projection of amplitude and phase information from the hindbrain to the midbrain of the African electric fish Gymnarchus niloticus. J Neurosci 18:7599-7611.

Kawasaki M, Guo Y-X (2002) Emergence of temporal-pattern sensitive neurons in the midbrain of weakly electric fish Gymnarchus niloticus. J Physiol (Paris) 96:531-537.

Kawasaki M, Maler L, Rose GJ, Heiligenberg W (1988) Anatomical and functional organization of the prepacemaker nucleus in gymnotiform electric fish: the accommodation of two behaviors in one nucleus. J Comp Neurol 276:113-131.

Keller CH (1988) Stimulus discrimination in the diencephalon of Eigenmannia: the emergence and sharpening of a sensory filter. J Comp Physiol [A] 162:747-757.

Keller CH, Heiligenberg W (1989) From distributed sensory processing to discrete motor representations in the diencephalon of the electric fish, Eigenmannia. J Comp Physiol [A] 164:565-576.

Keller CH, Maler L, Heiligenberg W (1990) Structural and functional organization of a diencephalic sensory-motor interface in the gymnotiform fish, Eigenmannia. J Comp Neurol 293:347-376.

Knudsen EI, Konishi M (1978) A neural map of auditory space in the owl. Science 200:795-797.

Koch C, Poggio T (1992) Multiplying with synapses and neurons. In: Single neuron computation (McKenna T, Davis J, Zornetzer SF, eds), pp 315-345. Boston: Academic.

Lissman HW (1958) On the function and evolution of electric organs in fish. J Exp Biol 35:156-191.

Margoliash D (1983) Acoustic parameters underlying the responses of songspecific neurons in the white-crowned sparrow. J Neurosci 3:1039-1057.

Mel BW (1992) NMDA-based pattern discrimination in a modeled cortical neuron. Neural Comput 4:502-517.

Mel BW (1993) Synaptic integration in an excitable dendritic tree. J Neurophysiol 70:1086-1101.

Metzner W (1993) The jamming avoidance response in Eigenmannia is controlled by two separate motor pathways. J Neurosci 13:1862-1878.

Misawa H, Suga N (2001) Multiple combination-sensitive neurons in the auditory cortex of the mustached bat. Hearing Res 151:15-29.

Moiseff A (1989) Bicoordinate sound localization by the barn owl. J Comp Physiol [A] 164:637-644.

Moiseff A, Konishi M (1983) Binaural characteristics of units in the owl's brainstem auditory pathway: precursors of restricted spatial receptive fields. J Neurosci 3:2553-2562.

Ohlemiller K, Kanwal JS, Butman JA, Suga N (1994) Stimulus design for auditory neuroethology: synthesis and manipulation of complex communication sounds. [A] ud Neurosci 1:19-37.

Olsen JF, Rauschecker JP (1992) Medial geniculate neurons in the squirrel monkey sensitive to combinations of components in a speciesspecific vocalization. Soc Neurosci [A]bstr 18:883.

Olsen JF, Suga N (1991a) Combination-sensitive neurons in the medial geniculate body of the mustached bat: encoding of relative velocity information. J Neurophysiol 65:1254-1273.

Olsen JF, Suga N (1991b) Combination-sensitive neurons in the medial geniculate body of the mustached bat: encoding of target range information. J Neurophysiol 65:1275-1296.

Partridge BL, Heiligenberg WF, Matsubara JA (1981) The neural basis of a sensory filter in the jamming avoidance response: no grandmother cells in sight. J Comp Physiol 145:153-168. 
Peña JL, Konishi M (2001) Auditory spatial receptive fields created by multiplication. Science 292:249-252.

Perrett DI, Rolls ET, Caan W (1982) Visual neurones responsive to faces in the monkey temporal cortex. Exp Brain Res 47:329-342.

Rose G, Heiligenberg WF (1986) Neural coding of difference frequencies in the midbrain of the electric fish Eigenmannia: reading the sense of rotation in an amplitude-phase plane. J Comp Physiol [A] 158:613-624.

Schnupp JWH, King AJ (2001) Neural processing: the logic of multiplication in single neurons. Curr Biol 11:R640-R642.

Srinivasan MV, Bernard GD (1976) A proposed mechanism for multiplication of neural signals. Biol Cybern 21:227-236.

Suga N (1994) Multi-function theory for cortical processing of auditory information: implications for single unit and lesion data for future research. J Comp Physiol [A] 175:135-144.

Suga N, O’Neill WE, Manabe T (1978) Cortical neurons sensitive to combinations of information-bearing elements of biosonar signals in the mustached bat. Science 200:778-781.

Sussman HM, Fruchter D, Hilbert J, Sirosh J (1998) Linear correlates in the speech signal: the orderly output constraint. Behav Brain Sci 21:241-299.

Torre V, Poggio T (1978) A synaptic mechanism possibly underlying directional selectivity to motion. Proc R Soc Lond B Biol Sci 202:409-416.

von der Emde G, Schwarz S, Gomez L, Budelli R, Grant K (1998) Electric fish measure distance in the dark. Nature 395:890-894. 